Thus if we know the solution of (13) in the form $b=b(x)$, we can write

and finally

$$
y=\exp \left(-\int b d x\right), \quad y_{1}=-b \exp \left(-\int b d x\right),
$$

$$
\eta=-b\{\phi(\xi)\} \exp \left[-\int b\{\phi(\xi)\} \phi^{\prime}(\xi) d \xi\right] .
$$

\title{
A Generalisation of a Theorem of Wolstenholme
}

\section{By HansRaj Gupta.}

$\S 1$. Chowla ${ }^{1}$ has generalised Wolstenholme's Theorem as follows:

$$
\sum_{a<\cdot p^{u}} \frac{1}{a} \equiv 0\left(\bmod . p^{2 u}\right), \quad p>3, u>0
$$

where $p$ denotes as usual a prime, and $<\cdot$ is used for "less than and prime to."

Denoting the greatest common divisor of two non-zero positive integers $n$ and $m$ by $\{n, m\}$, I here prove that

$$
\lambda . \sum_{a<\cdot n} \frac{1}{a} \equiv 0\left(\bmod . n^{2}\right),
$$

where $\lambda=\left\{n^{2}, \frac{12}{l}\right\}$, and $l=1,2$, or 4 , as defined later.

§2. Elsewhere ${ }^{2}$ I have shown that

$$
\begin{aligned}
\sum_{i=1}^{n-1} i^{s-2}=\frac{n^{(s-1)}}{s-1}+ & G(-s+2,1) \cdot \frac{n^{(s-2)}}{s-2}+\ldots+G(-k, s-k-1) \cdot \frac{n^{(k)}}{k} \\
& +\ldots+G(-2, s-3) \cdot \frac{n^{(2)}}{2},
\end{aligned}
$$

where $n^{(k)}=n(n-1)(n-2) \ldots(n-k+1) ; s>2$;

and $\quad(k-2) ! G(-k, s-k-1)=\sum_{j=0}^{k-2}\left\{(-1)^{j}\left(\begin{array}{c}k-2 \\ j\end{array}\right)(k-j-1)^{s-3}\right\}, \quad s>k$.

Evidently $\frac{n^{(k)}}{k} \equiv 0(\bmod . n)$, except when $k \mid n$, and is either 4 or a prime, 2,3 or $>3$. 
xii

In fact, when $k \mid n$ and is not a prime $>3$,

$$
\{k, 6\} \cdot \frac{n^{(k)}}{k} \equiv 0(\bmod . n) \text {. }
$$

In $[2 \cdot 1]$, let $n=p \geqq 3, s>2$, and $s-2 \neq 0(\bmod . p-1)$.

Then $\sum_{i=1}^{p-1} i^{s-2} \equiv \sum_{i=1}^{p-1} i^{r}(\bmod . p)$, where $s-2 \equiv r(\bmod . p-1), 1<r<p-1$, $\equiv 0(\bmod . p)$, since no $k \leqq r+1$ divides $n=p$.

In $[2 \cdot 2]$, let $k=p \geqq 3, s>2$, and $s-2 \neq 0(\bmod . p-1)$;

Then $(p-2) ! G(-p, s-p-1)=\sum_{j=0}^{p-2}\left\{(-1)^{j}\left(\begin{array}{c}p-2 \\ j\end{array}\right)(p-j-1)^{s-3}\right\}$,

$$
\begin{aligned}
& \equiv(-1)^{s-3} \sum_{j=0}^{p-2}(j+1)^{s-2},(\bmod . p) \\
& \equiv \quad 0 \quad \text { (mod. } p)
\end{aligned}
$$

Hence $G(-p, s-p-1) \equiv 0(\bmod . p), s>p \geqq 3, s-2 \neq 0(\bmod . p-1)$. [2.5] In view of $[2 \cdot 3]$ and $[2 \cdot 5]$, we have

$$
\{n, P\} \cdot \sum_{i=1}^{n-1} i^{s-2} \equiv 0(\bmod . n)
$$

where $P$ denotes the product of all $p^{\prime} \mathrm{s} \geqq 2$, which satisfy the congruence

$$
s-2 \equiv 0(\bmod . p-1) \text {. }
$$

$\S 3$. Proof of [1].

[1] is equivalent to $\frac{\lambda}{2} \sum_{a<\cdot n}\left\{\frac{1}{a}+\frac{1}{n-a}\right\} \equiv 0\left(\bmod . n^{2}\right)$;

or to

$$
\frac{\lambda}{2} \sum_{a<\cdot n} \frac{1}{a^{2}} \equiv 0(\bmod . n) \text {. }
$$

Let $n=p^{u} m$, where $p$ is a prime, $u \geqq 1$, and $\{m, p\}=1$.

Then $[3 \cdot 1]$ is equivalent to

$$
\frac{\lambda}{2} \phi(m) \cdot \sum_{a<\cdot p^{u}} a^{-2} \equiv 0\left(\bmod . p^{u}\right), \text { for each } p \mid n \text {. }
$$

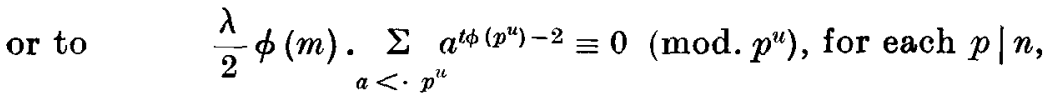
since $\quad a^{\phi\left(p^{u}\right)} \equiv 1\left(\bmod . p^{u}\right), \quad$ by Fermat's Theorem.

Let $t$ be an integer such that $t \phi\left(p^{u}\right)-2 \geqq u$.

Then $\sum_{a<\cdot p^{u}} a^{t \phi\left(p^{u}\right)-2} \equiv \sum_{a=1}^{p^{u}-1} a^{t \phi\left(p^{u}\right)-2}\left(\bmod \cdot p^{u}\right)$. 
Hence [1] is equivalent to

$$
\frac{\lambda}{2} \phi(m) \cdot \sum_{a=1}^{p^{n}-1} a^{t \phi\left(p^{u}\right)-2} \equiv 0\left(\bmod \cdot p^{u}\right), \text { for each } p \mid n .
$$

Now since $t \phi\left(p^{u}\right)-2 \equiv 0(\bmod . p-1)$, only if $p=2$ or 3 , [3.2] holds if $\frac{\lambda}{2} \phi(m) \equiv 0(\bmod .\{n, 6\})$.

When $n=2^{u}, u \geqq 1$, then $\phi(m)=1$ and $\lambda=4=\left\{n^{2}, 12\right\}$.

When $n=2^{u} m, u \geqq 0,\{m, 2\}=1$, if $\phi(m) \equiv 0(\bmod .2)$, then $\lambda=\{n, 6\}=\left\{n^{2}, 6\right\}$.

When $n=2^{u} m, u \geqq 0,\{m, 2\}=1$, if $\phi(m) \equiv 0(\bmod .4)$, then $\lambda=\{n, 3\}=\left\{n^{2}, 3\right\}$.

Hence if

$$
n=2^{u} N, \quad\{N, 2\}=1, \quad u \geqq 0 ;
$$

then $\lambda=\left\{n^{2}, \frac{12}{l}\right\}$, where $\phi(N)=l(\bmod .4), 1 \leqq l \leqq 4$.

\title{
REFERENCES.
}

1. S. Chowla, "A Teneralisation of a Theorem of Wolstenholme," The Muthematics Student, Vol. 1 (1933), pp. 140-141. See also Journal of the London Mathematical Society, Vol. 5 (1930), pp. 158-160.

2. Hansraj Gupta, "Operations with $\Delta$ on G-Functions." (In Press.)

\section{Note on the Summation of Finite Algebraic Series}

\author{
By J. A. Macdonald.
}

In order that an algebraic series of a finite number of terms may be summed in simple form, there must exist a difference equation satisfied by the terms. But, owing to the fact that no general method of finding this equation is given in textbooks, the beginner does not acquire confidence in summation until his experience is wide enough to include all of the usual types of such series; and even then he may come to the conclusion that the finding of the difference equation is no more than a lucky chance. It is therefore proposed in the following notes to show that the ordinary types of summable terminating series can be reduced to one, and to find a single general expression for the sum. It will also be shown that the criterion of summability is the "convergency ratio." 